\title{
Deficiency in Induction but Not Expression of LTP in Hippocampal Slices from Young Rats
}

Dezhi Liao $^{1,2}$ and Roberto Malinow ${ }^{1,3}$

${ }^{1}$ Cold Spring Harbor Laboratory

Cold Spring Harbor, New York 11724

${ }^{2}$ Department of Physiology and Biophysics

University of Iowa

Iowa City, Iowa 52245

\section{Abstract}

In this study we examine developmental changes between postnatal day (PND) 4 and 14 in synaptic transmission and plasticity in the CA1 region of hippocampal slices. We confirm previous results that tetanus-induced long-term potentiation (LTP) in field recordings is diminished in slices from younger animals. LTP in whole-cell current-clamp recordings is also diminished in younger animals. However, robust LTP can be induced in young animals if sufficient postsynaptic depolarization is provided during LTP induction. Furthermore, we find differences in synaptic transmission between PND 4 and 14, suggesting that the depolarization during tetanic stimulation in young tissue is ineffective to produce LTP. These results indicate that the smaller potentiation in field recordings in slices from younger animals is attributable to insufficient postsynaptic depolarization during LTP induction rather than a defect in expression mechanisms.

\section{Introduction}

Long-term potentiation (LTP), as characterized in the CA1 region of the hippocampus, is thought to have a role in learning and memory (Bliss and Collingridge 1993; Bannerman et al. 1995; Barnes 1995). Similar cellular mechanisms may also have critical roles during the development of the central nervous system (Cline et al.

\footnotetext{
${ }^{3}$ Corresponding author.
}

1987; Constantine-Paton et al. 1990). Both types of neuronal plasticity are activity-dependent (Archer et al. 1982; Constantine-Paton et al. 1990; Bliss and Collingridge 1993); both are thought to require Hebbian association of pre- and postsynaptic activity (Malinow and Miller 1986; Collingridge and Bliss 1987; Gustafsson et al. 1987; Fregnac et al. 1988; Greuel et al. 1988) and the activation of $N$-methyl-D-aspartate (NMDA) receptors (Cline et al. 1987; Collingridge and Bliss 1987). Recently, LTP was linked to critical period plasticity in the primary somatosensory cortex (Crair and Malenka 1995) and the primary visual cortex (Kirkwood et al. 1995). In these two studies, robust LTP was seen early in neocortical development. LTP in the hippocampus, however, may behave differently. Previous reports have indicated that hippocampal LTP is either difficult to generate (Harris and Teyler 1984; Muller et al. 1989) or not possible (Dudek and Bear 1993; Bolshakov and Siegelbaum 1995) in rats $<8$ days old.

It is not clear why there is less LTP in the hippocampus in younger animals. In general terms, one or more of the steps involved in the induction, expression, or maintenance of LTP may be defective (see Discussion). For example, sufficient depolarization during induction is necessary to generate LTP, and this may be more difficult to achieve in young animals. Alternatively, the calcium/calmodulin-dependent protein kinase II (CaMKII), an enzyme important to generate LTP, shows a developmentally controlled expression pattern that begins during the first postnatal week (Kelly et al. 1987; Scholz et al. 1988). A third possibility is that synaptic potentiation is already maximally saturated in the hippocampus of young rats (Bolshakov and Siegelbaum 1995).

Excitatory synapses in CA1 hippocampus, as in most of the regions studied in the vertebrate LEARNING \& MEMORY 3:138-149 (c) 1996 by Cold Spring Harbor Laboratory Press ISSN1072-0502/96 \$5.00

$$
\begin{array}{llllllll|c}
L & E & A & R & N & I & N & G & \boldsymbol{Q} \\
\mathbf{1 3 8} & M & E & M & O & R & Y
\end{array}
$$


LTP IN HIPPOCAMPUS OF VERY YOUNG RATS

central nervous system, transmit through the actions of glutamate on AMPA ( $\alpha$-amino-3-hydroxy5-methyl-4-isoxazolepropionate) and NMDA-type glutamate receptors (Collingridge et al. 1983; Collingridge and Lester 1989). On binding transmitter, these receptors have markedly different biophysical properties. AMPA receptors can conduct at hyperpolarized potentials, whereas NMDA receptors require depolarized potentials (Mayer et al. 1984). These receptors also have different roles in plasticity. Transient NMDA receptor activity is required to trigger LTP, whereas AMPA receptors are used in the expression of LTP (Collingridge and Bliss 1987). Although both receptor types are expressed on individual neurons (Petralia and Wenthold 1992; Good et al. 1993; Eshhar et al. 1993; Martin et al. 1993; Hollmann and Heinemann 1994; Petralia et al. 1994; Siegel et al. 1994, 1995 ), the distribution of receptors on individual synapses is less clear. Recently, we and others provided electrophysiological evidence for the existence of synapses that transmit with only NMDA receptors (Kullman 1994; Isaac et al. 1995; Liao et al. 1995; Durand et al. 1996). In our previous study we estimated that $\sim 60 \%$ of synapses were of this type, with the remainder containing both functional AMPA receptors and NMDA receptors. The fraction of synapses with only NMDA receptors may be important to the induction of LTP, as synaptic regions may fail to depolarize sufficiently during tetanic stimulation if they contain no AMPA type receptors.

In this study, we have investigated developmental changes of LTP in rat hippocampal CA1 region as measured with extracellular field, wholecell voltage-clamp and whole-cell current-clamp recordings. Consistent with previous studies, LTP is diminished in the early stages of development as measured with field and current-clamp recordings. However, robust LTP was generated when postsynaptic depolarization was provided directly during LTP induction in whole-cell voltage-clamp recordings. This suggests that the small LTP in young rats is attributable to insufficient postsynaptic depolarization during the induction protocol. In support of this, we find that synaptic transmission in baseline conditions and during tetanic stimulation produces different depolarization at different ages. The results indicate that the expression mechanisms for LTP and the biochemical components required for LTP induction downstream of NMDA receptor activation exist at the ages examined (postnatal day 5 to 14).

\section{Materials and Methods}

BRAIN SLICE PREPARATION AND RECORDING

Hippocampal slices $(400 \mu \mathrm{m})$ were prepared with a chopper or a vibratome from 4- to 14-dayold rats as described previously (Malinow and Tsien 1990). The slices were submerged and superfused with a bath solution containing $119 \mathrm{~mm}$ $\mathrm{NaCl}, 2.5 \mathrm{~mm} \mathrm{KCl}, 2.5 \mathrm{~mm} \mathrm{CaCl}, 1.3 \mathrm{~mm} \mathrm{MgCl}$, $26.2 \mathrm{~mm} \mathrm{NaHCO}, 1 \mathrm{~mm} \mathrm{NaH} \mathrm{PO}_{4}, 11 \mathrm{~mm}$ glucose, and gassed with $95 \% \mathrm{O}_{2}$ and $5 \% \mathrm{CO}_{2}$. Picrotoxin $(100 \mathrm{~mm})$ was added to block $\mathrm{GABA}_{\mathrm{A}}(\gamma$-aminobutyric acid) inhibitory postsynaptic currents. CA3 and CA1 regions were separated surgically to prevent epileptiform activity. Synaptic transmission was elicited by passing a brief $(<200 \mu \mathrm{sec})$ current pulse through an extracellular glass-stimulating electrode (filled with $2 \mathrm{M} \mathrm{NaCl}$ ) placed in $\mathrm{CA1}$ stratum radiatum $\sim 50 \mu \mathrm{m}$ from $S$. pyramidale.

Whole-cell recordings were obtained under visual guidance from cells two to three layers below the surface with a patch electrode (2-4 M $\Omega$ tip resistance) at a temperature of $24-28^{\circ} \mathrm{C}$. Series resistance was monitored and remained constant during an experiment (5-30 $\mathrm{M} \Omega$ ). The internal solution contained $100 \mathrm{~mm} \mathrm{CsMeSO}, 40 \mathrm{~mm}$ HEPES, 2 mм NaATP, $0.3 \mathrm{~mm}$ GTP, $5 \mathrm{~mm} \mathrm{MgCl}$, 5 mM glutathione, and $0.2 \mathrm{~mm}$ EGTA ( $\mathrm{pH} 7.2$ with $\mathrm{CsOH}$ ). In some experiments, $100 \mathrm{~mm}$ caesium gluconate was used instead of $100 \mathrm{~mm} \mathrm{CsMeSO}{ }_{3}$. No obvious difference was noticed between these two compounds in our whole-cell recordings. In current-clamp experiments, $100 \mathrm{~mm}$ potassium gluconate was used. Unless stated otherwise, holding potential was kept constant at a level between $-60 \mathrm{mV}$ and $-70 \mathrm{mV}$, and the stimulation frequency was kept at $1 / 6 \mathrm{~Hz}$ or $1 / 3 \mathrm{~Hz}$. In some experiments (Fig. 7, below), the stimulus level was decreased by small amount $(0.05 \mathrm{~V})$ until reaching a level that produced only failures for 75-100 trials after obtaining whole-cell recordings. That stimulus level is defined as a subminimal stimulus. In all voltage-clamp experiments, LTP was induced by pairing $2-\mathrm{Hz}$ presynaptic stimulation for $75 \mathrm{sec}$ with postsynaptic depolarization $(-10 \mathrm{mV})$. In all current-clamp experiments, LTP was induced by a tetanus protocol that consisted of 10 trains (separated from each other by $3 \mathrm{sec}$ ) of nine stimuli at $100 \mathrm{~Hz}$.

In field excitatory postsynaptic potential (EPSP) recordings, an extracellular glass recording electrode (filled with the above bath solution) was

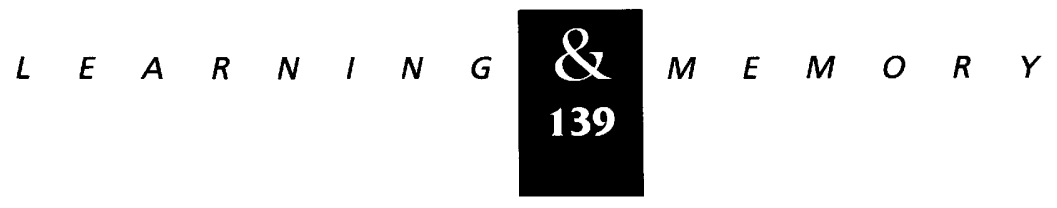


placed in CA1 stratum radiatum about 100-200 $\mathrm{mm}$ from an extracellular glass-stimulating electrode (filled with $2 \mathrm{M} \mathrm{NaCl}$ ). Unless stated otherwise, stimulation frequency was kept at 1/30 $\mathrm{Hz}$. LTP was induced by a tetanus protocol that consisted of five trains (separated by $30 \mathrm{sec}$ ) of nine stimuli at $100 \mathrm{~Hz}$.

\section{WHOLE-CELL SYNAPTIC AMPLITUDE MEASUREMENT AND DISTRIBUTION ESTIMATE}

Individual excitatory postsynaptic current (EPSC) peak amplitudes were determined by averaging the current over a fixed 1 - to 4-msec window encompassing the peak amplitude and subtracting the average current during a window prior to the stimulus (see Fig. 5, below). For a given experiment, the same windows were used on recorded sweeps in all experimental conditions. In some experiments, an additional 10-msec window placed 40-msec after the peak was used to estimate NMDA current at a depolarized potential $(+55 \mathrm{mV})$. The same windows placed during a period with no stimulus gave an estimate of the baseline noise. Density estimates of EPSC amplitudes in an epoch were generated by summing all Gaussian kernels with a standard deviation onehalf of the noise, each centered about an EPSC amplitude value on the $x$-axis (amplitude axis) (Silverman 1986; Malinow 1991; Liao et al. 1992). A similar method was used to estimate the noise density distribution. Such a method will overestimate the width of noise density distribution by $10 \%$. Let noise S.D. (standard deviation) $=1$, Gaussian kernel S.D. $=0.5$; therefore, the noise variance $=1$, Gaussian kernel variance $=0.25$; therefore, estimated noise distribution variance $=1+$ $0.25=1.25$, its S.D. $=(\text { variance })^{1 / 2}=1.12$.

\section{FRACTION OF POSTSYNAPTICALLY SILENT SYNAPSES}

The failure rate was quantified by measuring the amplitude of events and noise and constructing amplitude distribution density estimates (Figs. 1B,C; see Fig. 8, below). The failure peak was located near zero amplitude and matched the distribution of the noise. The proportion of synaptic failures for an epoch of responses was estimated by doubling the fraction of responses with amplitude less than the location of the failure peak. We estimate the fraction of release occurring at pure NMDA synapses by comparing the failure rate at
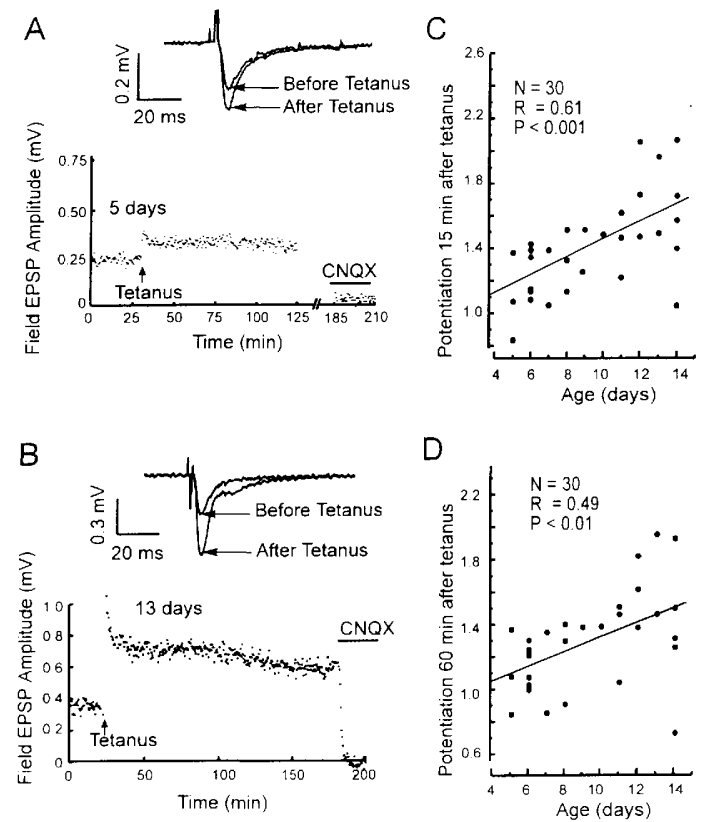

Figure 1: Tetanus-induced LTP of field EPSP in CA1 hippocampus is smaller in younger rats. (A) A small amount of LTP is induced in a hippocampal slice from a 5-day-old rat (a48s). (Top) Average field EPSP responses before ( 60 consecutive trials) and after ( 100 consecutive trials) tetanus. An average of responses obtained in 10 mM CNQX was digitally subtracted from raw traces to generate above averages. (Bottom) Field EPSP amplitude plotted vs. time in the same experiment. Arrow denotes delivery of tetanic protocol (see Materials and Methods). CNQX (10 $\mathrm{mm})$ applied where indicated, blocks responses. (B) Similar plots as $A$ from another experiment with larger LTP in a 13-day-old rat (a52o). (C,D) Potentiation of field EPSP amplitudes at 15 and 60 min posttetanus plotted vs. animal age. Synaptic potentiation is correlated positively with animal age $(C: n=30$, $\mathrm{R}=0.61, P<0.001 ; D: n=30, \mathrm{R}=0.49, P<0.01$ ).

depolarized $\left(F_{d}\right)$ and hyperpolarized $\left(F_{h}\right)$ potentials. We assume that the average probability of release $(P r)$ at releasing sites containing only NMDA receptors or mixed AMPA and NMDA receptors is similar. This assumption is reasonable given the observation that the NMDA to AMPA ratio in synaptic currents remains constant when the probability of release is modified 16-fold (Perkel and Nicoll 1993). The failure rates at depolarized and hyperpolarized potentials are approximated by $\mathrm{F}_{\mathrm{h}}=(1-P r)^{n_{\mathrm{AN}}}$ and $\mathrm{F}_{\mathrm{d}}=(1-$ $\operatorname{Pr})^{\left(n_{\mathrm{AN}}+n_{\mathrm{N}}\right)}$, where $n_{\mathrm{AN}}$ is the number of releasing sites containing both AMPA and NMDA receptors and $n_{\mathrm{N}}$ is the number of releasing sites containing only NMDA receptors. Rearrangement yields the fraction of pure NMDA synapses: $n_{\mathrm{N}} /$ 
$\left(n_{\mathrm{AN}}+n_{\mathrm{N}}\right)=1-\left(\ln \mathrm{F}_{\mathrm{h}} / \ln \mathrm{F}_{\mathrm{d}}\right)$. This is the same equation obtained by assuming a low probability of release (Hessler et al. 1993; Rosenmund et al. 1993) as described previously (Liao et al. 1995). This estimate will be affected in part by the recording noise. More noise will lead to counting events (real positive amplitude) as failures. Because the noise is greater at depolarized potentials, this leads to an overestimate of failures at depolarized potentials, minimizing the difference in failures at depolarized and hyperpolarized potentials. There was no systematic increase in noise with age, arguing against this as an artefactual basis for the age-dependent change in pure NMDA transmission.

\section{FIELD EPSP AMPLITUDE MEASUREMENT AND INPUT-OUTPUT CURVE}

Individual field EPSP peak amplitudes were determined by averaging the current over a fixed 1- to 4-msec window encompassing the peak amplitude and subtracting a baseline estimate. For a given experiment, the same windows were used on recorded sweeps in all experimental conditions. Stimulus artifact and fiber volley were estimated after $10 \mathrm{~mm}$ CNQX (6-cyano-7-nitroquinoxaine-2,3-dione) was applied at the end of most experiments, and were subtracted from each recorded field EPSP response. Experiments in which the baseline transmission drifted more than $15 \%$ (the early half vs. the late half) were excluded. At the beginning of most field EPSP experiments, we varied the stimulating intensity (input) at several levels, and measured the resulting field EPSP response (output). The stimulating intensity started at a level where no responses were observed until it reached a maximal level. Input-output lines were drawn for the linear part of the input/output curve by the standard linear regression method (Fig. 6A, below). The stimulation threshold was determined by calculating the intersection between an input-output curve and the $x$-axis (stimulation intensity). Thereafter the stimulation intensity was set midpoint between the threshold and the maximal level.

\section{Results}

INDUCTION OF LTP AT DIFFERENT AGES DURING EARLY POSTNATAL DEVELOPMENT

A number of previous studies have looked at LTP during development (Harris and Teyler 1984;
Muller et al. 1989; Dudek and Bear 1993; Bolshakov and Siegelbaum 1995). The general finding is that little or no LTP is possible at postnatal day (PND) $<8$. We repeated these experiments, measuring the field EPSP and inducing LTP with a strong tetanic protocol that contains five bursts (separated by $30 \mathrm{sec}$ ) of nine stimuli at $100 \mathrm{~Hz}$ (see Materials and Methods). Although we could see some persistent potentiation at young ages (Fig. 1A), the magnitude of LTP was clearly much larger at older ages (Figs. $1 \mathrm{~A}-\mathrm{D} ; \boldsymbol{n}=30, \mathrm{R}=\mathbf{0 . 4 9}$, $P<0.01$ at $60 \mathrm{~min}$ after tetanus).

We wished to determine whether transmission found in slices from younger animals lacked components necessary to express LTP, or whether there were aspects of LTP induction that were lacking. Specifically, we wished to test whether the amount of depolarization during LTP induction was insufficient in young slices. We tested this hypothesis directly by determining whether delivery of depolarizing current through a whole-cell recording electrode during LTP induction was sufficient to produce LTP in slices from young animals. We conducted a number of experiments on slices from different aged animals using whole-cell recordings in the voltage-clamp configuration (Fig. 2). After gaining whole-cell access from CA1 neurons, cells were clamped at $-60 \mathrm{mV}$ and synaptic transmission was elicited (See Materials and Methods). After a baseline period (5-10 $\mathrm{min}$ ), the postsynaptic membrane was depolarized to -10 $\mathrm{mV}$ and synaptic transmission was delivered at 2 $\mathrm{Hz}$ for $75 \mathrm{sec}$. Following this induction protocol, the postsynaptic membrane was repolarized to $-60 \mathrm{mV}$ and synaptic transmission resumed. As shown in Figure 2A, pairing presynaptic activity with postsynaptic depolarization produced synaptic potentiation in slices from 7-day-old rats. Pooling all experiments from 5- to 9-day-old animals, we saw clear evidence for LTP (Fig. 2C). We saw no evidence that pairing-induced LTP was greater at PND 10-14 compared with PND 4-9 (no correlation at Fig. $2 \mathrm{D}, n=24, \mathrm{R}=0.05, P>0.8$; see Fig. 5 for comparison with other types of recordings).

These results clearly show that expression of LTP is possible in slices from PND 5-9 rats. We wished to determine if the LTP observed in young animals required NMDA receptor function. We conducted the same protocol as above in the presence of $100 \mu \mathrm{M}$,L-2-amino-5-phosphonovaleric acid (DL-APV), an inhibitor of NMDA receptors. Under these conditions, LTP was blocked completely, indicating that the LTP observed in slices

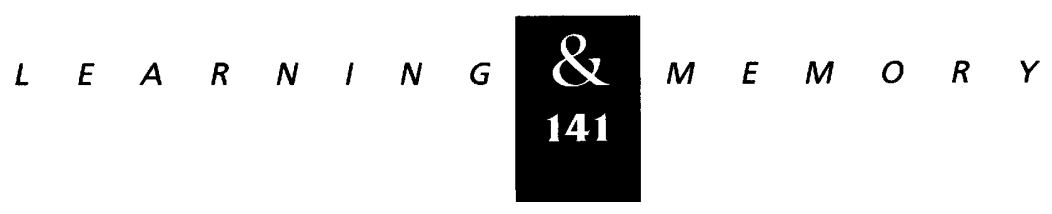




\section{Liao and Malinow}

Figure 2: Robust LTP can be induced in very young rats by pairing depolarization with synaptic transmission. (A) LTP in slice from a 7-day-old rat (a47a). (Top) Fifteen consecutive sweeps before (left) and after (right) pairing. (Middle) EPSC amplitude plotted vs. time. Delivery of a pairing protocol for LTP induction is indicated (see Materials and Methods). Large solid circles denote 10-point averages. Broken line denotes the average amplitude of baseline transmission. (Bottom) Series resistance plotted vs. time. $(B)$ Similar plots as $A$ from another experiment with a 14-day-old rat. Note the amount of potentiation in 7- and 14day-old animals is similar. (C) Statistical analysis of 23 whole-cell LTP experiments (5-9 days old). For each experiment, the average of responses in the first half of baseline period (base1) is assigned a value of 1 . There is no significant difference between the first (base1) and second (base2) half of baseline, indicating stability of transmission. Synaptic transmission is significantly potentiated at $3 \mathrm{~min}(n=23$; average age $=6.9$ days old), $15 \min (n=17 ; 7.0$ days old), $30 \mathrm{~min}\left(n=12 ; 7.3\right.$-days old), and $60 \mathrm{~min}\left(n=5 ; 7.2\right.$ days old) after a pairing protocol. $\left({ }^{* *}\right) P<0.01 ;\left({ }^{*}\right) P<0.05$; paired t-test. (D) Synaptic potentiation at $30 \mathrm{~min}$ after pairing plotted vs. animal age in 24 experiments. There is no significant correlation.

from PND 6-8 behaves like conventional LTP from older animals and depends on NMDA receptor function (Fig. 3). We further wished to determine if the ability to get LTP in young animals was attributable to the depolarization provided during induction, rather than some peculiarity of wholecell recording (wash-out of inhibitory substance, etc.). We therefore conducted a series of experiments with whole-cell recordings in currentclamp configuration. In these experiments, LTP was induced by delivering 10 bursts ( separated by $3 \mathrm{sec}$ ) of 9 tetanic stimuli at $100 \mathrm{~Hz}$ after a baseline period (5-10 $\mathrm{min}$ ). No depolarization was provided by the recording electrode. Under these conditions, LTP was significantly greater in slices from older animals, with little LTP in slices from young animals (Fig. 4, positive correlation at $\mathrm{E}$; $n=20, \mathrm{R}=0.54, P<0.02$; see Fig. 5 for comparison with other types of recordings).

\section{POSSIBLE MECHANISMS PRODUCING DEPOIARIZING DRIVE DURING TETANIC STIMULUS}

The results shown indicate that LTP is possible in younger animals if sufficient depolarization is provided during its induction. Is there less depolarization during synaptic transmission and tetanic stimulation in slices from young animals? We failed to find an age-related difference in a number of parameters examined. The initial absolute amplitudes of transmission as measured with field or current-clamp recordings could not account for the difference in LTP at different ages. Although there was a tendency for responses from younger animals to be smaller in the baseline period for field recordings ( $n=28, \mathrm{R}=0.3, P=0.11$ ), the amplitude of the initial response did not correlate significantly with the magnitude of potentiation ( $n=28, \mathrm{R}=0.18, P=0.37)$. Furthermore, in current-clamp recordings there was no positive correlation between initial amplitude and age ( $n=24$, $\mathrm{R}=-0.3, P=0.13$ ). Additionally, we have measured synaptic facilitation by measuring the ratio of the peak amplitude during a tetanus to the amplitude of baseline transmission. We found no significant correlation between synaptic facilitation and animal age $(n=28, \mathrm{R}=0.3, P>0.1)$.

Other measures, however, suggest that some aspects of depolarization may be different at different ages. Using field recordings, we constructed input-output curves (Fig. 6) and saw clear differ-

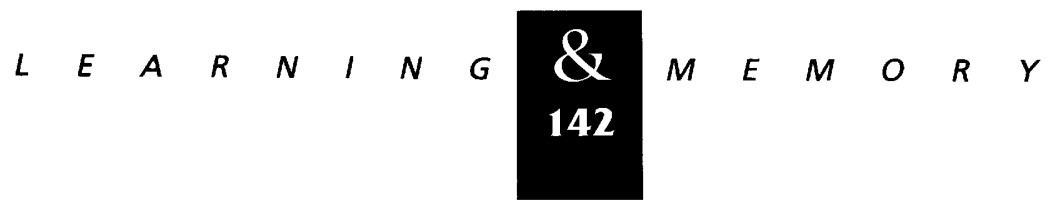


A

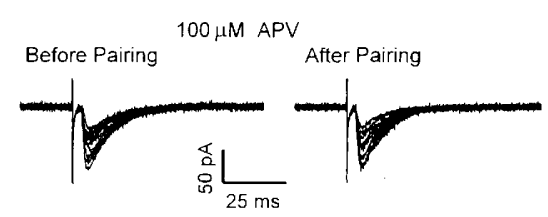

B
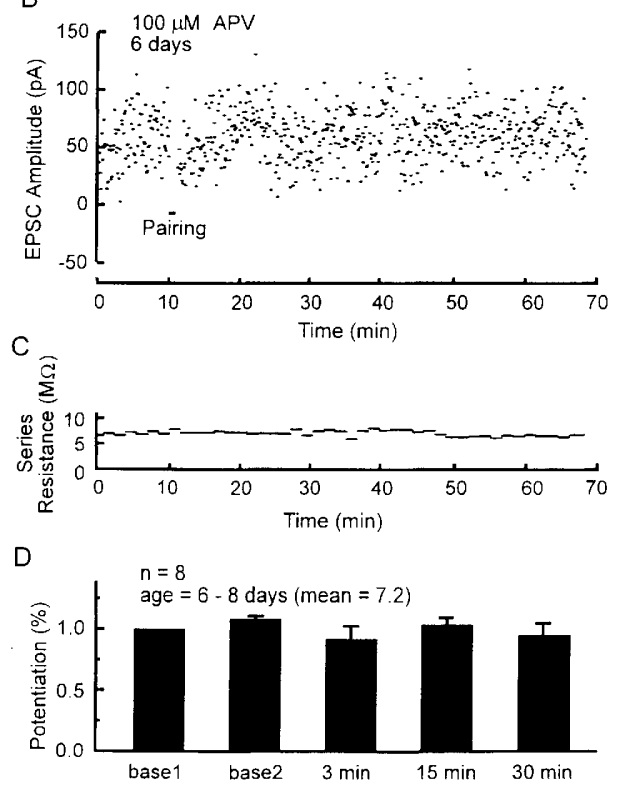

Figure 3: LTP in slices from young rats is blocked by APV. (A) Twelve consecutive sweeps before (left) and after (right) pairing in $100 \mu \mathrm{M}$ DL-APV (a49t, 6 days old). (B) EPSC amplitude plotted vs.time from the same experiment as in $A$. (C) Series resistance plotted vs. time from the same experiment as $A$ and $B$. (D) Summary analysis of eight experiments ( $6-8$ days old). Baseline normalization as in Fig. 2. Synaptic transmission was not significantly changed at 3, 15, or $30 \mathrm{~min}$ after pairing (for all intervals, $P>0.05$, paired $t$-test).

ences in these curves in rats from different ages. The slope of the I/O curve as well as the threshold stimulus for obtaining a response were positively and negatively correlated, respectively, with age (Fig. 6; see also Harris and Teyler 1984). This suggests that recruitment of synaptic drive, as assessed by an extracellular stimulus, is less in younger animals.

One possible mechanism producing smaller local depolarizations is if a large fraction of synapses contain no functional AMPA receptors. Recently, we have described transmission mediated by pure NMDA receptor function in hippocampal neurons (Liao et al. 1995). This can be demonstrated clearly by reducing the stimulus intensity so that few axons are stimulated. In some cases, one can observe all failures at hyperpolarized po-
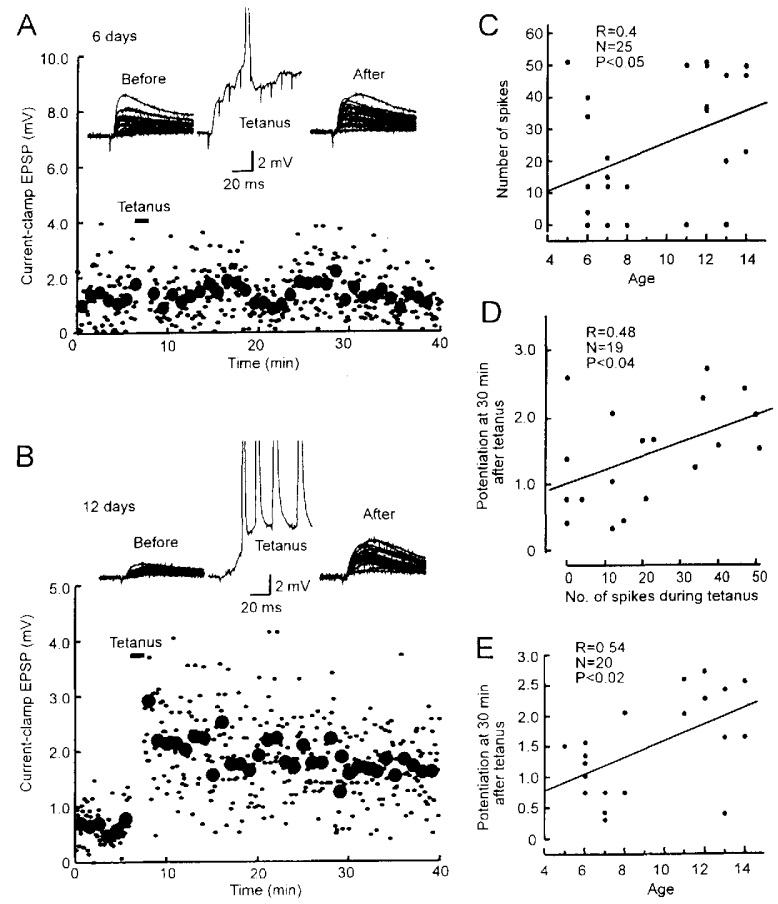

Figure 4: LTP recorded in current-clamp is age-dependent. (A) Whole-cell recording from a 6-day-old rat (a57c). (Top) Twenty consecutive individual sweeps before (left) and after (right) tetanus, with a representative response to a train of nine tetanic stimuli (middle). Only one action potential is generated during this train. (Bottom) Whole-cell EPSP amplitude plotted vs. time. Delivery of tetanus is indicated. (B) Similar plots as $A$ in another experiment from a 12-day-old rat (a57n). Note that more spikes were evoked during a tetanic train than in $A$. (C) Total number of action potentials evoked during tetanus protocol plotted vs. animal age. More spikes are evoked in more mature rats $(n=25, R=0.4$, $P<0.05$ ). (D) Potentiation of current-clamp EPSP amplitudes at $30 \mathrm{~min}$ post-tetanus plotted vs. number of spikes during tetanus protocol. Synaptic potentiation is correlated positively with number of spikes $(n=19, R=0.48$, $P<0.04)$. (E) Potentiation of current-clamp EPSP amplitudes at $30 \mathrm{~min}$ post-tetanus plotted vs. animal age. Synaptic potentiation is correlated positively with animal age $(n=20, \mathrm{R}=0.54, P<0.02)$.

tentials with clear outward synaptic current at depolarized potentials. This can be blocked completely by APV, indicating pure NMDA receptor mediated transmission (Fig. 7A). From such subminimal transmission, LTP can be elicited if depolarization is provided by the recording electrode during LTP induction (Fig. 7B-D). The fraction of transmission mediated by pure NMDA receptor function can be quantified by comparing the failure rates at depolarized and hyperpolarized poten-

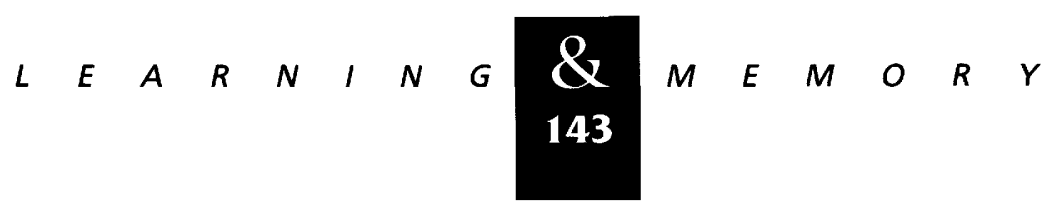


A

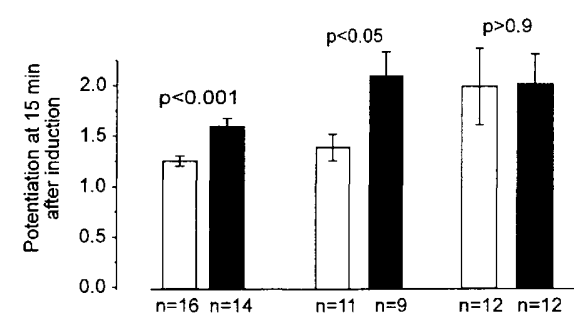

B

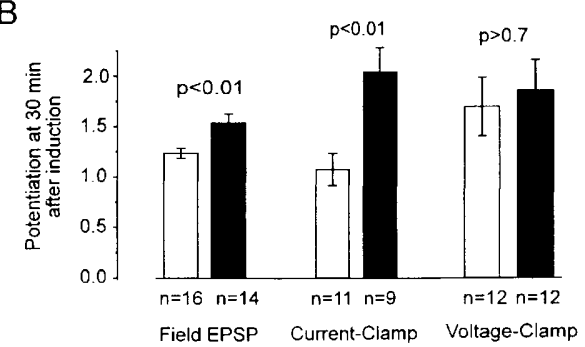

Figure 5: Comparison of synaptic potentiation obtained under different types of recordings for 4- to 9-dayold rats (open bars) and 10- to 14-day-old rats (solid bars). (A) Synaptic potentiation at $15 \mathrm{~min}$ after a LTP. inducing protocol (see Materials and Methods), respectively, in field EPSP (left), whole-cell current-clamp (middle) and whole-cell voltage-clamp recordings (right). Note significant difference in the amount of synaptic potentiation between 4- to 9-day-old rats and 10to 14-day-old rats in both field EPSP and whole-cell current-clamp recordings $(P<0.001$ and $P<0.05$, respectively). However, no significant difference is found between these two groups of rats in voltage-clamp recordings $(P>0.9)$. (B) A similar plot as $A$ at $30 \mathrm{~min}$ after a LTP-inducing protocol.

tials (Liao et al. 1995). This provides an estimate of the quantal content at synapses with only NMDA receptor function, relative to the quantal content at synapses with AMPA receptor function. We characterized this property in slices from different aged animals. Transmission was elicited by a weak stimulus whose intensity was set so that $\sim 50-90 \%$ of responses were failures at hyperpolarized potentials. The failure rate was quantified by estimating the density distribution of EPSC peak amplitude and noise (see Materials and Methods). In agreement with our previous studies (Liao et al. 1995), we saw more failures when the postsynaptic membrane was held at a hyperpolarized potential than at a depolarized potential, indicating the existence of pure NMDA transmission (Fig. 8A-C). Although there was some scatter in the data, there was a clear decrease in the estimated fraction of transmission mediated purely by NMDA receptors
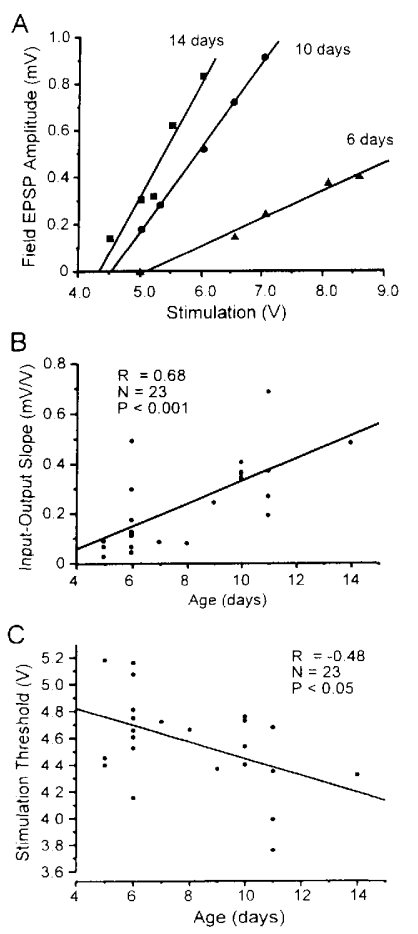

Figure 6: Field EPSP in the hippocampus of a younger rat has a lower input-output slope and a higher stimulation threshold. (A) Field EPSP amplitude plotted vs. stimulation intensity in three representative experiments (a47w: 14-day-old; a47s: 10-day-old; a48f: 6-day-old). (B) Input-output slope plotted vs. animal age shows positive correlation $(n=23, P<0.001)$. (C) Stimulation threshold (see Materials and Methods) plotted vs. animal age shows positive correlation $(n=23, P<0.05)$.

during development from PND 4 to PND 14 (Fig. $8 \mathrm{D} ; n=34, \mathrm{R}=-0.52, P<0.005)$. The corollary to this finding is that the fraction of release at synapses with AMPA receptor function is low at early ages, increasing from PND 4 to PND 14. This suggests the possibility that a local synaptic region may lack sufficient functional AMPA receptors required for effective postsynaptic depolarization to induce LTP.

In addition to the comparison of quantal contents of these two receptor types, we measured the ratio of net synaptic AMPA current to net synaptic NMDA current (Fig. 9). This measure will take into account not only the quantal content but also the quantal size of these two components. Interestingly, whereas the quantal components showed developmental changes, the ratio of net synaptic currents mediated by the two receptor types showed no significant changes (Fig. 9C; $n=50 ; \mathrm{R}=-0.04 ; P>0.8)$. This was true for the

$$
\begin{array}{lllllllllllllllll} 
& E & A & R & N & I & N & G & \begin{array}{l}
\bigotimes \\
144
\end{array} & M & E & M & O & R & Y
\end{array}
$$




\section{LTP IN HIPPOCAMPUS OF VERY YOUNG RATS}

A

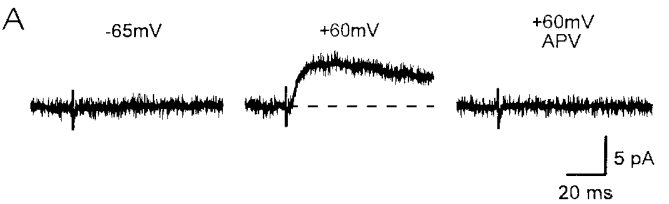

B
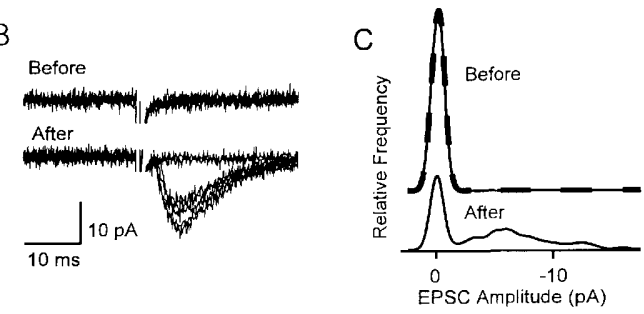

D

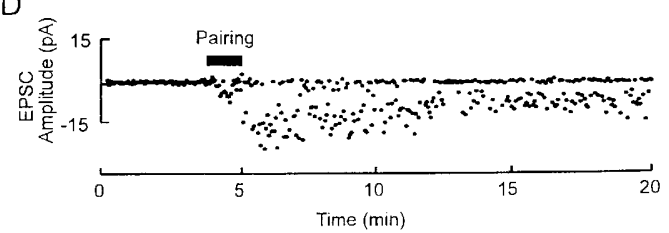

Figure 7: Pure NMDA-receptor mediated transmission and LTP at silent synapses. (A) Average of 50 consecutive responses to a subminimal stimulus (see Materials and Methods) with holding potential $-65 \mathrm{mV}$ (left), $+55 \mathrm{mV}$ (middle), and + $55 \mathrm{mV}$ after application of 100 $\mu \mathrm{M}$ DL-APV (right) in an individual experiment (a51b; 6-day-old rat). (B) Eight consecutive responses to a subminimal stimulus before (top) and after (bottom) delivery of a pairing protocol (see text); holding potential is -65 $\mathrm{mV}$ (a30e). (C) EPSC amplitude distributions before (top) and after pairing (bottom) in the same experiment as $B$. The noise distribution (thick broken line) fits the EPSC amplitude distribution before pairing, indicating the absence of responses. (D) EPSC amplitude plotted vs. time for the experiment in $B$ and $C$; pairing protocol delivered were indicated.

currents obtained with minimal stimulation to estimate failure rates $(n=35 ; R=-0.11 ; P>0.5)$ as well as for currents generated with stronger stimulus $(n=15, \mathrm{R}=-0.12 ; P>0.7)$. These results suggest the observed age-dependent differences in LTP are not attributable to a larger NMDA current in more mature rats.

Are there other measures indicating inadequate depolarization during LTP induction in slices from young animals? There has been interest recently in the role of action potential generation in relation to synaptic integration and plasticity (Jaffe et al. 1992; Spruston et al. 1995; Magee and Johnston 1995). With whole-cell current-clamp recordings, we examined the number of spikes generated during a tetanus for different age animals (Fig. 4A,B, insets). Plotting the total number of spikes generated during the tetanic protocol delivered to induce LTP versus age of animal, we found a positive correlation (Fig. $4 \mathrm{C} ; n=25$; $\mathrm{R}=0.4 ; P<0.05$ ). This indicates that fewer action potentials are generated during tetanic stimulation in neurons from slices of younger animals. Furthermore, there was a significant positive correlation between the number of spikes generated during LTP induction and the magnitude of potentiation at 5,15 , and $30 \mathrm{~min}$ after a tetanus ( $5 \mathrm{~min}: \boldsymbol{n}=22$, $P<0.001 ; 15 \min : n=21, P<0.04 ; 30 \min : n=19$, $P<0.04$; Fig. 4D).

\section{Discussion}

In this study we have attempted to answer one question: Why is there less LTP in tissue from 1-week-old rats compared with 2-week-old rats? In agreement with previous studies, we find that the magnitude of LTP as measured in field or currentclamp recordings increases between PND 4 and 14 (Figs. 1 and 4). Although LTP at 1 week is smaller than at 2 weeks, there is nevertheless clear LTP in young animals with field recordings (e.g., Fig. 1A). Several proposals have been made to explain this developmental progression. One possibility is that the biochemical machinery involved in modifying synapses is developmentally regulated and is lacking in 1-week-old animals. Another possibility is that synapses initially have a saturated amount of LTP and that during development synapses get weaker and therefore allow LTP to be expressed (Bolshakov and Siegelbaum 1995).

To address this issue we have considered the steps hypothesized to occur in the generation of LTP. A tetanic stimulus recruits activity in presynaptic axons in a repetitive manner. Transmitter is released producing spatio-temporal summation of EPSPs and action potentials. This depolarization allows opening of postsynaptic NMDA receptors and entry of calcium. These events are considered transient and therefore part of the steps involved in LTP induction. These events are thought to cause the biochemical machinery of synapses to be modified in such a manner that transmission is enhanced permanently. The cellular mechanisms that keeps the biochemical machinery in this modified state are considered to be responsible for the maintenance of LTP. The specific effect of this modified machinery is considered to be the site of LTP expression. We have asked if the small amount of LTP in young tissue is attributable to a defi-

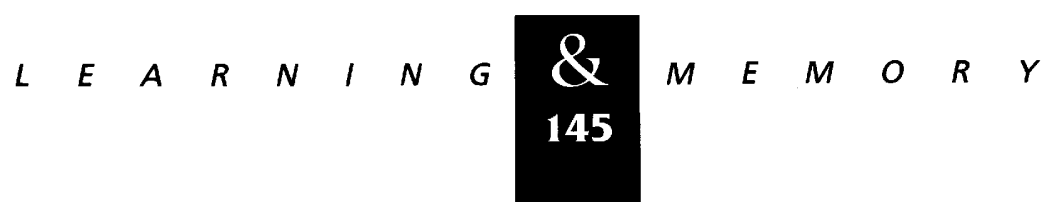


Figure 8: More postsynaptically silent transmission at developmentally earlier stages in CA1/subiculum rat hippocampus. (A) Amplitude of evoked EPSCs plotted vs. trials for an individual experiment; holding potential for different epochs is shown. (B) The amplitude distributions of EPSCs at hyperpolarized $(-65 \mathrm{mV}$, thick solid line) and depolarized (+55 mV, thin solid line) potentials from a 7-dayold rat (a43f). Density estimates of noise at $-65 \mathrm{mV}$ (thick broken line) and +55 $\mathrm{mV}$ (thin broken line) fit the failure peaks; failure rates given in parentheses. The difference in failure rate can be explained if $91 \%$ synapses are postsynaptically silent at hyperpolarized potentials. (C) Amplitude distributions as in $B$ for transmission in a 14-day-old rat (a42i). Difference in failure rate indicates $61 \%$ synapses are
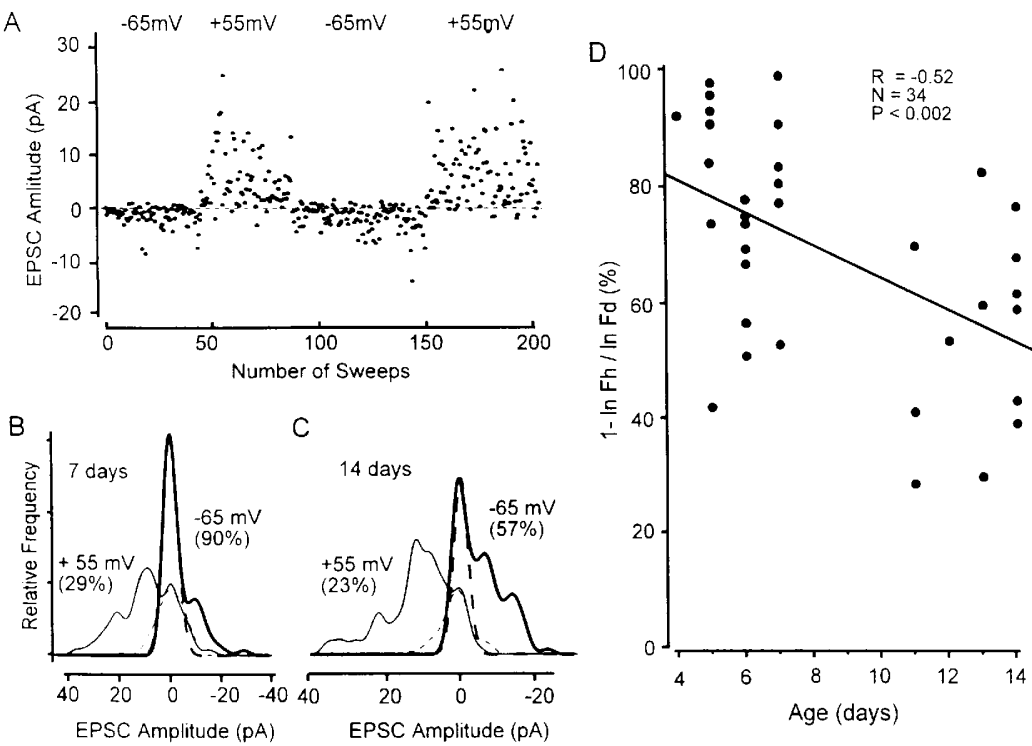
postsynaptically silent. (D) Percentage of postsynaptically silent synapses ( $1-\ln \mathrm{Fh} / \mathrm{ln} \mathrm{Fd}$ ) plotted vs. animal age (see Materials and Methods). Linear regression analysis indicates probability of correlation being attributable to chance is $<0.002$.

ciency in the induction, maintenance, or expression of LTP.

Our data argues for a defect in the induction and not the maintenance or expression of LTP. Specifically, our results support the view that in young tissue, a tetanic stimulus does not provide the necessary depolarization to induce LTP. The inability to generate LTP in young slices is not simply because we produced smaller baseline responses in slices from young animals. There appears to be a more subtle aspect of depolarization during tetanic stimulus, rather than the amplitude of baseline transmission, that determines potentiation. It is possible that the amount of synaptic drive in any local dendritic region could be lower in younger animals. This is one common way to interpret three different findings concerning basal transmission in younger animals: the shallower $\mathrm{I} / \mathrm{O}$ slope, the higher stimulus threshold necessary to produce a response, and the higher fraction of transmission mediated by pure NMDA receptors. If a large local depolarization is necessary to open synaptic NMDA receptors, then this interpretation would predict less NMDA receptor activation during tetanic stimulus and less LTP. Furthermore, we see fewer action potentials generated during a tetanus in young animals and a significant correlation between the number of action potentials generated and the amount of LTP. These data provide evidence that the amount and nature of depolar- ization provided by tetanic stimulation is significantly different in younger animals and this difference contributes to the smaller amount of LTP found in these animals.

The strongest support of the hypothesis that an insufficient depolarization during LTP induction is responsible for the diminished LTP in young animals is the finding that we can get robust LTP in slices from young animals if depolarizing current is provided during LTP induction (Figs. 2 and 5). This finding clearly indicates that the biochemical machinery sufficient for expression and maintenance of LTP is present in 1-week-old animals. This finding also counters directly a recently proposed hypothesis regarding the development of LTP. It was proposed (Bolshakov and Siegelbaum 1995) that the diminished LTP in young animals was attributable to a saturation of expression mechanisms. Specifically, it was proposed that the probability of transmitter release (Pr) is close to 1 (i.e., saturated) in young animals and that LTP is caused by an increase in Pr. Furthermore, it was proposed that as the animal matures there is a decrease in $\mathrm{Pr}$, and thereby allowing for the expression of LTP by increases in Pr. However, we clearly see LTP with both field (Fig. 1) and whole-cell recordings (Figs. 2 and 4) that is blocked by APV (Fig. 3) at ages where it was claimed that $P_{\mathrm{r}}$ is 1 and LTP was saturated. This finding is not consistent with the claims that: (1) 

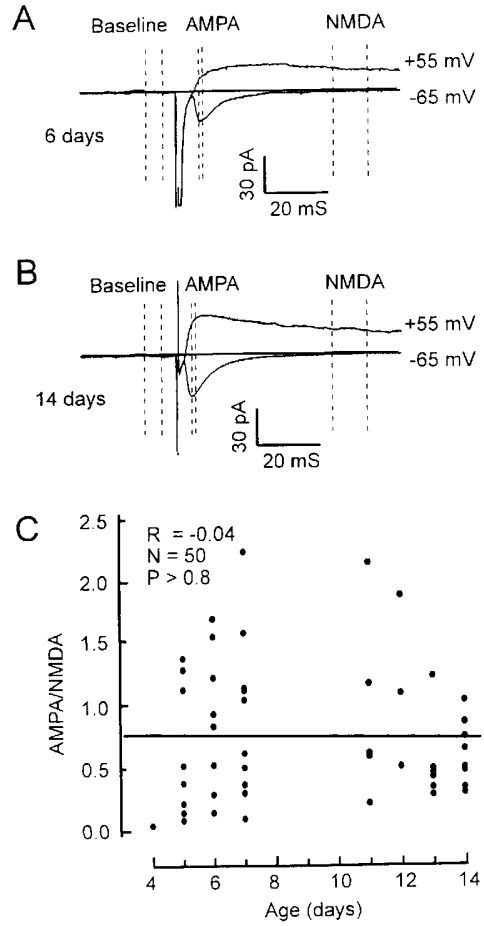

Figure 9: The ratio of synaptic AMPA current/NMDA current is not correlated with animal age. (A) Averaged EPSCs at depolarized (top, $+55 \mathrm{mV}$ ) and hyperpolarized (bottom, $-65 \mathrm{mV}$ ) potential. Broken lines represent windows selected for estimating the base line, AMPA current (at hyperpolarized potential) and NMDA current (at depolarized potential) (a42e, 6 days old). (B) Records similar to $A$ from a 14-day-old rat (a43a). (C) The ratio of AMPA current/NMDA current plotted vs. animal age. No correlation is found between the ratio and animal age $(n=50, R=-0.04, P>0.8)$.

$\operatorname{Pr}=1$ in young animals and (2) LTP is attributable to an increase in $\mathrm{Pr}$. At least one of these two statements is inconsistent with our results. Alternative explanations for the observations of the Boshakov and Siegelbaum study (1995) are that (1) their experimental conditions biased toward selection of a small subgroup of synapses that were not representative of the whole population of synapses that we see with stronger stimulation or (2) their experimental conditions biased against the induction (rather than expression) of LTP.

As in a previous study, we have estimated the fraction of synaptic events acting purely on NMDA receptors by comparing the failure rate at depolarized and hyperpolarized potentials (Liao et al. 1995). With this estimate, we find that there is a higher prevalence of release at pure NMDA synapses earlier in development. At PND 4-5 almost
$80-90 \%$ of release appears to occur at synapses that transmit with only NMDA receptors. By PND 13-14 this fraction is $\sim 50 \%$. A recent study (Durand et al. 1996) also reached a similar conclusion regarding the age-dependent prevalence of pure NMDA-mediated transmission using a different stimulation protocol. However, unlike our estimate, their estimate of pure NMDA receptor mediated transmission depends on the assumption that (1) they can reliably stimulate a single presynaptic fiber and (2) that a single fiber makes a single contact. These assumptions are not universally accepted.

The decrease in pure NMDA-receptor mediated transmission during development parallels what we have described previously to occur during LTP (Liao et al. 1995). This observation supports the possibility that an LTP-like process occurs during development, converting pure NMDA receptor synapses to synapses with mixed AMPA and NMDA receptor function. Pairing-induced LTP cannot account for all synaptic changes observed during development, however. For instance, pairing-induced LTP produces an increase in the ratio of synaptic current mediated by AMPA receptors relative to NMDA receptors (Liao et al. 1995). This differs from what we find during development of these neurons, where no significant change is detected in the AMPA/NMDA ratio in synaptic currents. It is notable that more comparable changes in AMPA and NMDA receptor-mediated currents can occur during tetanus-induced LTP (Tsien and Malinow 1990; Bashir et al. 1991; O'Connor et al . 1995). How do we interpret the lack of change in AMPA/NMDA ratio along with the change in the fraction of transmission occurring at pure NMDA synapses? The latter is a measure of the relative quantal contents of synapses using these different receptors, whereas the former is a measure of the quantal content and quantal size. These observations can be reconciled if a change in quantal size of the AMPA and NMDA synapses offsets the change in quantal contents. This could be if quantal size of synapses with AMPA receptors decrease during development or if the quantal size of NMDA increases with development.

In conclusion, we find that in slices from young animals LTP can be induced if sufficient depolarization is provided during its induction. We also find that during tetanically induced LTP there are some aspects of depolarization that are diminished in slices from young animals. These along with other factors under developmental 
control are likely to limit the induction of LTP in young animals. It remains to be seen whether there are mechanisms used in vivo that obviate this limitation.

\section{Acknowledgments}

The publication costs of this article were defrayed in part by payment of page charges. This article must therefore be hereby marked "advertisement" in accordance with 18 USC section 1734 solely to indicate this fact.

\section{References}

Archer, S.M., M.W. Dubin, and L.A. Stark. 1982. Abnormal development of kitten retino-geniculate connectivity in the absence of action potentials. Science 217: 743-745.

Bannerman, D.M., M.A. Good, S.P. Butcher, M. Ramsay, and R.G.M. Morris. 1995. Distinct components of spatial learning revealed by prior training and NMDA receptor blockade. Nature 378: 182-186.

Barnes, C.A. 1995. Involvement of LTP in memory: Are we "searching under the street light"? Neuron 15: 751-754.

Bashir, Z.I., S. Alford, S.N. Davies, A.D. Randall, and G.L. Collingridge. 1991. Long-term potentiation of NMDA receptor-mediated synaptic transmission in the hippocampus. Nature 349: 156-158.

Bliss, T.V. and G.L. Collingridge. 1993. A synaptic model of memory: Long-term potentiation in the hippocampus. Nature 361: 31-39.

Bolshakov, V.Y. and S.A. Siegelbaum. 1995. Regulation of hippocampal transmitter release during development and long-term potentiation. Science 269: 1730-1734.

Cline, H.T. and M. Constantine-Paton. 1989. NMDA receptor antagonists disrupt the retinotectal topographic map. Neuron 3: 413-426.

Cline, H.T., E. Debski, and M. Constantine-Paton. 1987. NMDA receptor antagonist desegregates eye-specific stripes. Proc. Natl. Acad. Sci. 84: 4342-4245.

Collingridge, G. and T. Bliss. 1987. NMDA receptors-their role in long-term potentiation. Trends Neurosci. 10: $288-293$.

Collingridge, G.L. and R.A.J. Lester. 1989. Excitatory amino acid receptors in the vertebrate central nervous system. Pharmacol. Rev. 40: 143-210.

Collingridge, G.L., S.J. Kehl, and H. Mclennan. 1983. Excitatory amino acids in synaptic transmission in the schaffer collateral-commissural pathway of the rat hippocampus. I. Physiol. 334: 33-46.

Constantine-Paton, M., H.T. Cline, and E. Debski. 1990. Patterned activity, synaptic convergence, and the NMDA receptor in developing visual pathways. Annu. Rev. Neurosci. 13: 129-154.

Crair, M.C. and R.C. Malenka. 1995. A critical period for long-term potentiation at thalamocortical synapses. Nature 375: $325-328$.

Dudek, S M. and M.F. Bear. 1993. Bidirectional long-term modification of synaptic effectiveness in the adult and immature hippocampus. J. Neurosci. 13: 2910-2918.

Durand, G.M., Y. Kovalchuk, and A. Konnerth. 1996. Long-term potentiation and functional synapse induction in developing hippocampus. Nature 381: 71-75.

Eshhar, N., R.S. Petralia, C.A. Winter, A.S. Niedzielski, and R.J. Wenthold. 1993. The segregation and expression of glutamate receptor subunits in cultured hippocampal neurons. Neuroscience 57: 943-964.

Fregnac, Y., D. Shulz, S. Thorpe, and E. Bienenstock. 1988. A cellular analogue of visual cortical activity in primary visual cortical plasticity. Nature 333: 367-370.

Good, P.F., G.W. Huntley, S.W. Rogers, S.H. Heinemann, and J.H. Morrison. 1993. The distribution of kainate receptor subunits GluR5/6/7 in primate hippocampus. Brain Res. 624: $347-353$.

Greuel, J.M., H.J. Luhmann, and W. Singer. 1988. Pharmacological induction of use-dependent receptive field modifications in the visual cortex. Prog. Neurobiol. 30: $171-191$.

Gustafsson, B., H. Wigstrom, W.C. Abraham, and Y.-Y. Huang. 1987. Long-term potentiation in the hippocampus using depolarizing current pulses as the conditioning stimulus to single volley synaptic potentials. J. Neurosci. 7: 774-780.

Harris, K.M. and T.J. Teyler. 1984. Development onset of long-term potentiations in area CA1 of the rat hippocampus. I. Physiol. 346: 27-48.

Hessler, N., A. Shirke, and R. Malinow. 1993. The probability of transmitter release at a mammalian central synapse. Nature 366: 569-572.

Hollman, M. and S. Heinemann. 1994. Cloned glutamate receptors. Annu. Rev. Neurosci. 17: 31-108.

Issac, J.T.R., R.A. Nicoll, and R.C. Malenka. 1995. Evidence for silent synapses: Implications for the expression of LTP. Neuron 15: 427-434.

Jaffe, D.B., D. Johnston, N. Lasser-Ross, J.E. Lisman, H. Miyakawa, and W.N. Ross. 1992. The spread of $\mathrm{Na}^{+}$spikes determines the pattern of dendritic $\mathrm{Ca}^{2+}$ entry into hippocampal neurons. Nature 357: 244-246.

Jahr, C.E. and C.F. Stevens. 1987. Glutamate activates multiple single channel conductances in hippocampal neurons. Nature 325: 522-525. 
Kelly, P.T., S. Shields, K. Conway, R. Yip, and K. Burgin. 1987. Developmental changes in calmodulin-kinase II activity at brain synaptic junctions: Alterations in holoenzyme composition. J. Neurochem. 49: 1927-1940.

Kirkwood, A., H.K. Lee, and M.F. Bear. 1995. Co-regulation of long-term potentiation and experience-dependent synaptic plasticity in visual cortex by age and experience. Nature 375: 328-331.

Kullman, D.M. 1994. Amplitude fluctuations of dual-component EPSCs in hippocampal pyramidal cells: Implications for long-term potentiation. Neuron 12: $1111-1120$.

Liao, D., A. Jones, and R. Malinow. 1992. Direct measurement of quantal changes underlying long-term potentiation in CA1 hippocampus. Neuron 9: 1089-1097.

Liao, D., N.A. Hessler, and R. Malinow. 1995. Activation of postsynaptically silent synapses during pairing-induced LTP in CA1 region of hippocampal slice. Nature 375: 400-404.

Magee, J.C. and D. Johnston. 1995. Synaptic activation of voltage-gated channels in the dendrites of hippocampal pyramidal neurons. Science 268: 301-304.

Malinow R. 1991. Transmission between pairs of hippocampal slice neurons: Quantal levels, oscillations, and LTP. Science 252: 722-724.

Malinow, R. and J.P. Miller. 1986. Postsynaptic hyperpolarization during conditioning reversibly blocks induction of long-term potentiation. Nature 320: 529-530.

Malinow, R. and R.W. Tsien. 1990. Presynaptic enhancement shown by whole-cell recording of long-term potentiation in hippocampal slices. Nature 346: 177-180.

Martin, L.J., C.D. Blackstone, A.I. Levey, R.L. Huganir, and D.L. Price. 1993. AMPA receptor subunits are differentially distributed in rat brains. Neuroscience 53: 327-358.

Mayer, M.L., G.L. Westbrook, and P.B. Guthrie. 1984. Voltage-dependent block by $\mathrm{Mg}^{2+}$ of NMDA responses in spinal cord neurones. Nature 309: 261-263.

Muller, D., M. Oliver, and G. Lynch. 1989. Developmental changes in synaptic properties in hippocampal neonatal rats. Dev. Brain Res. 49: 105-114.

O'Connor, J.J., M.J. Rowan, and R. Anwyl. 1995. Tetanically induced LTP involves a similar increase in the AMPA and NMDA receptor components of the excitatory postsynaptic current: Investigations of the involvement of mGlu receptors. J. Neurosci. 15: 2013-2020.

Perkel, D.J. and R.A. Nicoll. 1993. Evidence for all-or-none regulation of neurotransmitter release: Implications for long-term potentiation. J. Physiol. 471: 481-500.

Petralia, R.S. and R.J. Wenthold. 1992. Light and electron immunocytochemical localization of AMPA-selective glutamate receptors in the rat brain. J. Comp. Neurol. 318: 329-354.

Petralia, R.S., N. Yokotani, and R.J. Wenthold. 1994. Light and electron microscope distribution of NMDA receptor subunit NMDAR1 in the rat nervous system using a selective anti-peptide antibody. J. Neurosci. 14: 667-696.

Rosenmund, C., J.D. Clements, and G.L. Westbrook. 1993. Nonuniform probability of glutamate release at a hippocampal synapse. Science 262: 754-757.

Siegel, S.J., N. Brose, W.G. Janssen, G.P. Gasic, R. Jahn, S.F. Heinemann, and J.H. Morrison. 1994. Regional, cellular, and ultrastructural distribution of the glutamate receptor subunit NMDAR1 in monkey hippocampus. Proc. Natl. Acad. Sci. 91: 564-568.

Siegel, S.J., W.G. Janssen, J.W. Tullai, S.W. Rogers, T. Moran, S.F. Heinemann, and J.H. Morrison. 1995.

Distribution of the excitatory amino acid receptor subunits GluR2(4) in monkey hippocampus and colocalization with subunits GluR5-7 and NMDAR1. J. Neurosci.

15: $2707-2719$.

Silverman, V. 1986. Density estimate for statistics and data analysis. Chapman \& Hall, New York, NY.

Scholz, K.W., C. Baitinger, H. Schulman, and P.T. Kelly. 1988. Developmental changes in $\mathrm{Ca}^{2+} /$ calmodulin-dependent protein kinase II in cultures of hippocampal pyramidal neurons and astrocytes. J. Neurosci. 8: 1039-1051.

Spruston, N., Y. Schiller, G. Stuart, and B. Sakmann. 1995. Activity-dependent action potential invasion and calcium influx into hippocampal CA1 dendrites. Science 268: $297-300$.

Tsien, R.W. and R. Malinow. 1990. Long-term potentiation: Presynaptic enhancement following postsynaptic activation of $\mathrm{Ca}(++)$-dependent protein kinases. Cold Spring Harbor Symp. Quant. Biol. 55: 147-159.

Received June 14, 1996; accepted in revised form July 30, 1996.

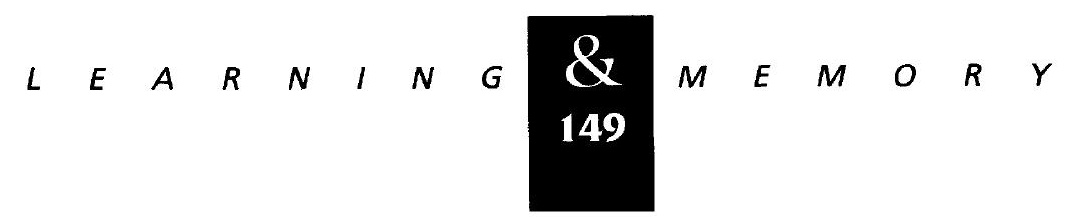




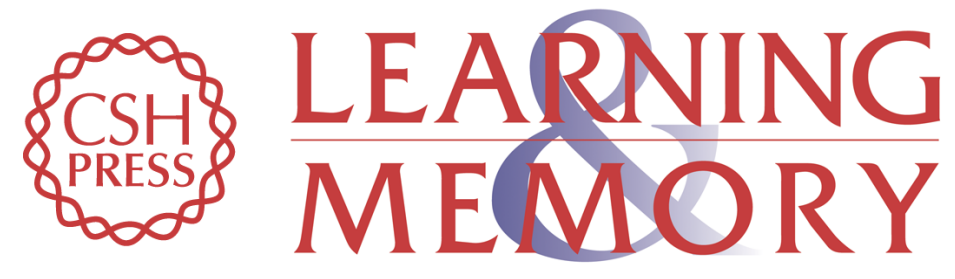

\section{Deficiency in induction but not expression of LTP in hippocampal slices from young rats.}

D Liao and R Malinow

Learn. Mem. 1996, 3:

References This article cites 48 articles, 15 of which can be accessed free at: http://learnmem.cshlp.org/content/3/2-3/138.full.html\#ref-list-1

License

Email Alerting

Receive free email alerts when new articles cite this article - sign up in the box at the Service top right corner of the article or click here. 